\title{
The Effects of Tamarindus Indica Seed Extract On Mice Kidney, Liver and Skin Following Envenomation with Daboai Russelli And Naja Kaouthia
}

Soraya Ismail ${ }^{1}$,Pakeer Oothuman ${ }^{1}$, Khin Maung Maung ${ }^{2}$

${ }^{1}$ Department of Basic Medical Sciences, Kulliyyah of Medicine, International Islamic University Malaysia

${ }^{2}$ Biochemistry Research Division, Department of Medical Research, Ministry of Health and Sport, Myanmar

Presenter: Soraya Ismail

Introduction: Tamarindus indica seed extract (TSE) has anti-snake-venom properties. This study examined the histological changes of kidney, liver and skin of mice envenomed with either Daboai russelli and Naja kaouthia and treated with TSE. Materials and Methods: ICR mice were used. Group 1 mice $(n=8)$ were given subcutaneous (SC) venom only of either species. Group $2(n=8)$ envenomed mice were treated with SC TSE $30 \mathrm{mg} / 20 \mathrm{~g}$ at the same site 30 minutes after envenomation with either species. Tissue samples were harvested at 24 hours and 1 week. Transversely cut kidney, biggest liver lobe, and skin sample from the venom injection site were fixed in 10\% formal-saline and stained with Haematoxylin-Eosin for light-microscopy analysis. Results: Group 1 mice exposed to D.russelli venom, all died within 48hours. Hence, no comparison was made between Groups 1 and 2 at 1 week. However histological comparison was made within Group 2 at 24 hours and 1 week. Kidney histological changes of envenomed mice showed mild nephropathy; liver showed inflammation and mild liver injury. Skin samples showed disruption of the skin architecture. These changes, worsened after 1 week. However, changes in the skin were unaltered after 1 week for N. kaouthia. When TSE $30 \mathrm{mg} / 20 \mathrm{~g}$ was administered, there was restoration of tissue architecture in mice envenomed with either species. Conclusion: When mice were exposed to $L D_{100}$ of either snake venoms, histological changes took place within 24 hours. This study showed that TSE $30 \mathrm{mg} / 20 \mathrm{~g}$ was able to restore tissue architecture within 24 hours. 